\title{
Synthesis of new pyrazolyl-2, 4-thiazolidinediones as antibacterial and antifungal agents
}

\author{
Deepak K Aneja ${ }^{1 *}$, Poonam Lohan', Sanjiv Arora', Chetan Sharma², Kamal R Aneja ${ }^{2}$ and Om Prakash ${ }^{3 \dagger}$
}

\begin{abstract}
Background: Thiazolidine-2, 4-diones (TZDs) have become a pharmacologically important class of heterocyclic compounds since their introduction in the form of glitazones into the clinical use for the treatment of type 2 diabetes. TZDs lower the plasma glucose levels by acting as ligands for gamma peroxisome proliferators-activated receptors. In addition, this class of heterocyclic compounds possesses various other biological activities such as antihyperglycemic, antimicrobial, anti-inflammatory, anticonvulsant, insecticidal, etc. TZDs are also known for lowering the blood pressure thereby reducing the chances of heart failure and micro-albuminuria in the patients with type 2 diabetes.
\end{abstract}

Results: We have described herein the synthesis of three series of compounds, namely, ethyl 2-((Z)-5-((3-aryl-1phenyl-1H-pyrazol-4-yl)methylene)-2, 4-dioxothiazolidin-3-yl)acetates (4), methyl 2-((Z)-5-((3-aryl-1-phenyl-1H-pyrazol4-yl)methylene)-2, 4-dioxothiazolidin-3-yl)acetates (5), and 2-((Z)-5-((3-aryl-1-phenyl-1H-pyrazol-4-yl)methylene)-2, 4dioxothiazolidin-3-yl)acetic acids (6). The compounds $\mathbf{4}$ and $\mathbf{5}$ were synthesized by Knoevenagel condensation between 3-aryl-1-phenyl-1H-pyrazole-4-carbaldehydes (1) and ethyl/methyl 2-(2, 4-dioxothiazolidin-3-yl)acetates (3, 2) in alcohol using piperidine as a catalyst. The resultant compounds $\mathbf{4}$ and $\mathbf{5}$ having ester functionality were subjected to acidic hydrolysis to obtain $\mathbf{6}$. All the new compounds were tested for their in vitro antibacterial and antifungal activity.

Conclusions: Knoevenagel condensation approach has offered an easy access to new compounds 4-6. Antimicrobial evaluation of the compounds has shown that some of the compounds are associated with remarkable antifungal activity. In case of antibacterial activity, these were found to be effective against Grampositive bacteria. However, none of the compounds were found to be effective against Gram-negative bacteria.

Keywords: thiazolidine-2, 4-dione, pyrazole, Knoevenagel condensation, antibacterial activity, antifungal activity

\section{Background}

Natural antibiotic compounds have become essential to current health care system, assisting and complementing the natural immune system against microbial pathogens. As conventional antibiotics are often abused to treat microbial infections, some microorganisms have developed tolerance to these antibiotics. Because of the appearance of antibiotic-resistant strains, the continuous development of novel efficient antibiotic agents is more crucial than ever [1-3]. So, the medical community faces a serious problem against infections caused by the

\footnotetext{
* Correspondence: dk_aneja@rediffmail.com

† Contributed equally

'Department of Chemistry, Kurukshetra University, Kurukshetra 136119,

Haryana, India

Full list of author information is available at the end of the article
}

pathogen bacteria and needs an effective therapy and search for novel antimicrobial agents. Synthetic organic chemistry has always been a vital part of highly integrated and multidisciplinary process of various drug developments. In this context, this study was designed to evaluate antimicrobial properties of new pyrazole derivatives containing thiazolidindiones.

Pyrazole derivatives are known to possess wide spectrum of pharmacological properties such as antibacterial [4-6], antifungal [7-9], antimicrobial [10-14], antidiabetic [15], herbicidal [16,17], antitumor [18-21], anti-anxiety [22], and as active pharmacophore in celecoxib (as COX-2 inhibitor) [23] and slidenafil citrate [24] (as cGMP specific phosphodiesterase type 5 inhibitor), etc. Pyrazoles play an essential role in biological active 
compounds and therefore represent an interesting template for medicinal chemistry.

On the other hand, thiazolidines are also known for their potential biological activities. The varied biological activities of rhodanines (2-thioxo-thiazolidin-4-one) and their analogs have been known from the beginning of twentieth century. Rhodanines and 2, 4-thiazolidinediones (TZDs) have become a pharmacologically important class of heterocyclic compounds since the introduction of various glitazone and epalrestat into clinical use for the treatment of type II diabetes and diabetic complications [25]. Several studies have been reported that TZDs have acquired much importance because of their diverse pharmaceutical applications such as antihyperglycemic [26], bactericidal [27], pesticidal [28], fungicidal [29], insecticidal [30], anticonvulsant [31], tuberculostatic [32], anti-inflammatory [33] etc.

Different possibilities of heterocyclic modifications with a wide spectrum of pharmacological propertiesare the most important grounds for investigation of this class of compounds. There have been many reports in literature depicting that the presence of heterocyclic moieties such as thiazole, pyrazole, flavone, chromone, sultam, and furan at fifth position proves to be more potent and efficacious than a simple aryl group [34-39]. Although there are not many TZDs fused to pyrazoles, a number of them are incorporated into a wide variety of therapeutically important compounds possessing a broad spectrum of biological activities. In a recent article, pyrazolyl-2, 4-TZDs have been reported as antiinflammatory and neuroprotective agents.

Motivated by these findings and in continuation of our ongoing efforts endowed with the discovery of nitrogen-containing heterocycles with potential chemotherapeutic activities [8,10,40-44], we disclose here the synthesis and investigations of antimicrobial activities of new pyrazolyl-2, 4-TZD.

\section{Results and discussion}

\subsection{Chemistry}

The synthetic route for the preparation of ethyl 2-((Z)5-((3-aryl-1-phenyl-1H-pyrazol-4-yl)methylene)-2, 4dioxothiazolidin-3-yl)acetates (4a-h), methyl 2-((Z)-5((3-aryl-1-phenyl-1H-pyrazol-4-yl)methylene)-2, 4-dioxothiazolidin-3-yl)acetates (5a-h), and 2-((Z)-5-((3-aryl-1phenyl-1H-pyrazol-4-yl)methylene)-2, 4-dioxothiazolidin-3-yl)acetic acids (6a-h) has been illustrated in Scheme 1. Initially, Knoevenagel condensation was carried out with equimolar ratio of ethyl 2-(2, 4-dioxothiazolidin-3-yl)acetate (3) and 1, 3-diphenyl-1H-pyrazole-4carbaldehyde (1a) in ethanol in presence of catalytic amount of piperidine by refluxing for 5-6 h. The usual work up of the reaction afforded the single product, ethyl 2-((Z)-2, 4-dioxo-5-((1, 3-diphenyl-1H-pyrazol-4-

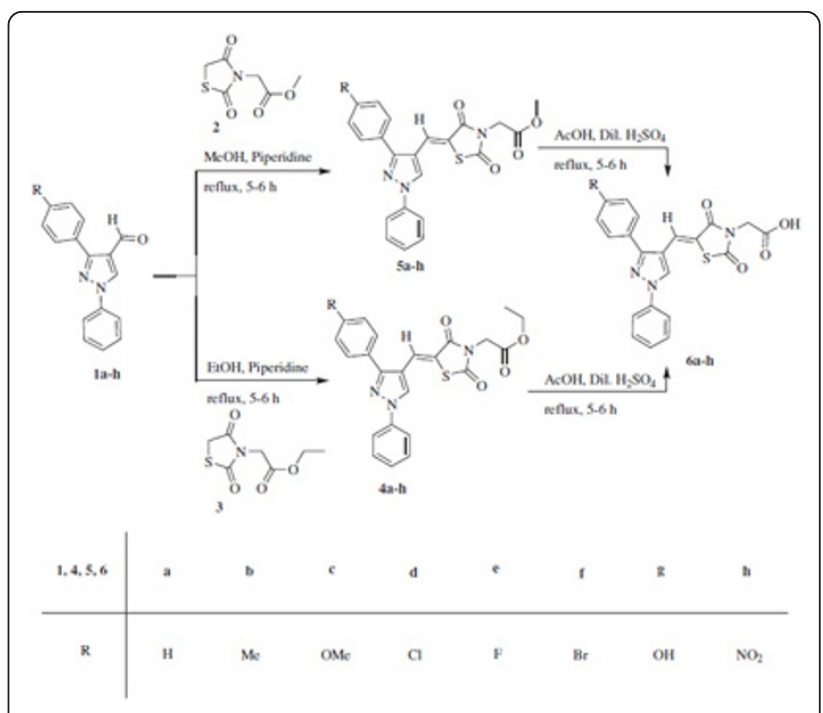

Scheme 1 Synthesis of pyrazolyl-2, 4-TZDs (4-6).

yl)methylene)thiazolidin-3-yl)acetate (4a) as yellow solid in $90 \%$ yield. Similar method was adopted for the preparation of $\mathbf{5 a}$ in methanol. The acid hydrolysis of $\mathbf{4 a}$ or $5 \mathbf{a}$ in acetic acid in the presence of dilute sulfuric acid under refluxing for 5-6 $\mathrm{h}$ gave the desired product 2((Z)-2, 4-dioxo-5-((1, 3-diphenyl-1H-pyrazol-4-yl)methylene)thiazolidin-3-yl)acetic acid (6a) in 94\% yield.

All other compounds $\mathbf{4 b}-\mathbf{h}, \mathbf{5 b}-\mathbf{h}$, and $\mathbf{6 b}-\mathbf{h}$ were prepared adopting the similar methodology. The physical data of all compounds 4-6 have been summarized in Table 1.

The structures of all compounds $\mathbf{4 a - h}, \mathbf{5 a}-\mathbf{h}$, and $\mathbf{6} \mathbf{a}-\mathbf{h}$ were established by the spectral (IR, NMR \{see additional files $1,2,3,4,5,6,7,8,9,10,11,12,13,14,15$, $16,17,18,19,20,21,22,23$ and 24\}, Mass) and elemental analysis. For example, IR spectrum of the compound 4a exhibited characteristic absorption bands at 1736 and $1690 \mathrm{~cm}^{-1}$ because of carbonyl groups of ester and TZD. The ${ }^{1} \mathrm{H}$ NMR spectrum of the product $4 \mathbf{a}$ (see additional files 1) showed three characteristic singlets at $\delta$ $8.213, \delta 7.963$, and $\delta 4.473$ because of $\mathrm{C}(5)-\mathrm{H}$ of pyrazole ring, $=\mathrm{CH}$ and $-\mathrm{NCH}_{2}$, respectively, apart from other aromatic signals. Besides these the aliphatic region also showed the characteristic quartet and triplet due to $-\mathrm{OCH}_{2} \mathrm{CH}_{3}$ at $\delta 4.248$ and $\delta 1.301$, respectively. The product $\mathbf{6 a}$ was characterized by careful comparison of the IR and ${ }^{1} \mathrm{H}$ NMR spectra (see additional file 17) with those of the 4a. An important characteristic feature in ${ }^{1} \mathrm{H}$ NMR spectrum of $\mathbf{6} \mathbf{a}$ was disappearance of the triplet and quartet in the aliphatic region which was present in the spectrum of $\mathbf{4 a}$.

The starting materials 3 -aryl-1-phenyl- $1 H$-pyrazole-4carbaldehydes $(\mathbf{1} \mathbf{a}-\mathbf{h})$ were prepared according to literature procedure involving Vilsmeier-Haack reaction of 
Table 1 Physical data of the compounds 4-6

\begin{tabular}{lll}
\hline Compounds & Yields (\%) & Melting points $\left({ }^{\circ} \mathbf{C}\right)$ \\
\hline $\mathbf{4 a}$ & 90 & $223-225$ \\
$\mathbf{4 b}$ & 92 & $225-227$ \\
$\mathbf{4 c}$ & 91 & $274-276$ \\
$\mathbf{4 d}$ & 92 & $248-250$ \\
$\mathbf{4 e}$ & 93 & $237-239$ \\
$\mathbf{4 f}$ & 93 & $258-260$ \\
$\mathbf{4 g}$ & 94 & $248-250$ \\
$\mathbf{4 h}$ & 95 & $231-233$ \\
$\mathbf{5 a}$ & 92 & $225-227$ \\
$\mathbf{5 b}$ & 94 & $233-235$ \\
$\mathbf{5 c}$ & 91 & $263-265$ \\
$\mathbf{5 d}$ & 93 & $248-250$ \\
$\mathbf{5 e}$ & 91 & $233-235$ \\
$\mathbf{5 f}$ & 92 & $269-271$ \\
$\mathbf{5 g}$ & 90 & $280-282$ \\
$\mathbf{5 h}$ & 93 & $240-242$ \\
$\mathbf{6 a}$ & 94 & $294-296$ \\
$\mathbf{6 b}$ & 93 & $300-302$ \\
$\mathbf{6 c}$ & 94 & $262-264$ \\
$\mathbf{6 d}$ & 93 & $280-282$ \\
$\mathbf{6 e}$ & 92 & $304-306$ \\
$\mathbf{6 f}$ & 90 & $288-290$ \\
$\mathbf{6 g}$ & 94 & $317-319$ \\
$\mathbf{6 h}$ & 91 & $287-288$ \\
\hline
\end{tabular}

various substituted acetophenone hydrazones using $\mathrm{POCl}_{3} / \mathrm{DMF}$ at $50-60^{\circ} \mathrm{C}$ for $4-5 \mathrm{~h}$ [45-47] and ethyl/ methyl 2-(2, 4-dioxothiazolidin-3-yl)acetates $(3,2)$ were prepared in multiple steps by alkylation of potassium salt of thiazolidine-2, 4-dione (TZDs) with appropriate alkyl 2-bromoacetate either in acetone at $50^{\circ} \mathrm{C}$ for $5 \mathrm{~h}$

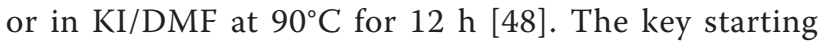
material 2, 4-TZD needed for this purpose was obtained in one step from equimolar amounts of chloroacetic acid and thiourea under ice cold condition. The white precipitate of 2-imino thiazolidine-4-one obtained was then acidified and refluxed with $\mathrm{HCl}$ for $12 \mathrm{~h}$ to get white crystals of 2, 4-TZD [49].

Although geometrical isomerism ( $E / Z$ isomers) was possible because of restricted rotation about the exocyclic $\mathrm{C}=\mathrm{C}$ bond of the pyrazolyl-2, 4-TZDs, all the derivatives prepared in this study were obtained exclusively in $Z$-form as confirmed by the analytical data. The ${ }^{1} \mathrm{H}$ NMR spectra of the pyrazolyl-2, 4-TZDs (see additional files $1,2,3,4,5,6,7,8,9,10,11,12,13,14,15,16,17$, $18,19,20,21,22,23,24)$ showed that the most characteristic olefinic proton $=\mathrm{CH}$ was deshielded more $(\delta=$ 7.3-7.6 ppm) as expected in $Z$-form, relative to the slightly shielded protons of the $E$-form $(\delta=6.2-6.3 \mathrm{ppm}$, in case of various other arylidene-2, 4-TZD). This deshielding of the olefinic proton is caused by the anisotropic effect exerted by the nearby carbonyl group of the 2, 4-TZDs in $Z$-isomer. Furthermore, the $Z$-isomers are thermodynamic more stable because of intramolecular hydrogen bond that can be formed between the hydrogen bond of $=\mathrm{CH}$ and oxygen atom in TZD $[50,51]$.

\subsection{Pharmacology}

\subsubsection{In vitro antifungal activity}

All the 24 compounds were tested for their in vitro antifungal activity against two fungi, namely, Aspergillus niger and Aspergillus flavus. Standard antibiotic, namely, Fluconazole, was used for comparison with antifungal activity shown by compounds $\mathbf{4 a - h}, \mathbf{5 a - h}$, and $\mathbf{6 a - h}$. A careful analysis of percentage mycelial growth inhibition revealed that almost all the newly synthesized compounds showed comparable antifungal activity with commercial antibiotics Fluconazole as shown in Table 2.

Compounds $\mathbf{4 b}$ and $\mathbf{4 e}$ showed maximum inhibition against $A$. niger (70\%) and A. flavus (67.7\%), respectively. Eleven compounds 4d, 4e, 4g, 5a, 5h, 6a, 6b, 6d, 6e, 6f, and $\mathbf{6 h}$ showed more than $60 \%$ inhibition against $A$. flavus in comparison to $77.7 \%$ of Fluconazole. Eleven compounds which showed more than $60 \%$ inhibition against

Table 2 In vitro antifungal activity of the compounds 4-6

\begin{tabular}{lll}
\hline Compounds & \multicolumn{2}{l}{ Mycelial growth of inhibition (\%) } \\
\cline { 2 - 3 } & A. flavus & A. niger \\
\hline 4a & 54.4 & 60.0 \\
4b & 54.4 & 70.0 \\
4c & 48.8 & 54.4 \\
4d & 61.1 & 65.5 \\
4e & 67.7 & 61.1 \\
4f & 55.5 & 62.5 \\
4g & 61.1 & 54.4 \\
4h & 48.8 & 58.8 \\
5a & 62.5 & 55.5 \\
5b & 48.8 & 54.4 \\
5c & 54.4 & 62.5 \\
5d & 55.5 & 61.1 \\
5e & 57.7 & 55.5 \\
5f & 67.7 & 62.5 \\
5g & 54.4 & 57.7 \\
5h & 61.1 & 54.4 \\
6a & 61.1 & 62.5 \\
6b & 63.3 & 61.1 \\
6c & 55.5 & 60.0 \\
6d & 61.5 & 62.5 \\
6e & 65.5 & 62.5 \\
6f & 65.5 & 61.1 \\
6g & 54.4 & 58.8 \\
6h & 61.1 & 60.0 \\
Fluconazole & 77.7 & 81.1 \\
\hline & &
\end{tabular}


A. niger are $\mathbf{4 b}, \mathbf{4 d}, \mathbf{4 e}, \mathbf{4 h}, \mathbf{5 c}, \mathbf{5 d}, \mathbf{6 a}, \mathbf{6 b}, \mathbf{6 d}, \mathbf{6 e}, \mathbf{6 f}$. After all, the compounds which showed more than $60 \%$ inhibition against both the pathogenic fungi are $\mathbf{4 a}, \mathbf{4 e}$, 6a, 6d, and 6e.

\subsubsection{In vitro antibacterial activity}

All the 24 compounds $\mathbf{4 a - h}, \mathbf{5 a}-\mathbf{h}$, and $\mathbf{6} \mathbf{a}-\mathbf{h}$ were tested in vitro for their antibacterial activity against two Grampositive bacteria, namely, Staphylococcus aureus (MTCC 96), Bacillus subtillis (MTCC 121) and two Gram-negative bacteria, namely, Escherichia coli (MTCC 1652), and Pseudomonas aeruginosa (MTCC 741) (Tables 3 and 4). Minimum inhibitory concentrations (MIC) of those compounds were determined which were showing activity in primary screening. Standard antibiotic, Ciprofloxacin, was used for comparison with antibacterial activity shown by the compounds $\mathbf{4 a - h}, \mathbf{5 a}-\mathbf{h}$, and $\mathbf{6 a}-\mathbf{h}$.

All compounds of the tested series showed variable antibacterial activity against Gram-positive bacteria. Three of the tested compounds $\mathbf{5 h}, \mathbf{6 a}$, and $\mathbf{6 h}$ exhibited good antibacterial activity against Gram-positive bacteria. However, none of the compounds showed activity against Gram-negative bacteria.

Table 3 In vitro antibacterial activity of the compounds

4-6

\begin{tabular}{|c|c|c|}
\hline \multirow[t]{2}{*}{ Compounds } & \multicolumn{2}{|c|}{ Diameter of the growth of zone inhibition $(\mathrm{mm})^{2}$} \\
\hline & S. aureus & B. subtilis \\
\hline $4 a$ & 15.6 & 16.3 \\
\hline $4 b$ & 16.3 & 15.0 \\
\hline $4 c$ & 15.3 & 14.6 \\
\hline $4 d$ & 14.3 & 14.6 \\
\hline $4 e$ & 13.6 & 14.0 \\
\hline $4 f$ & 16.6 & 17.6 \\
\hline $4 \mathrm{~g}$ & 15.0 & 15.6 \\
\hline $4 \mathrm{~h}$ & 19.0 & 17.0 \\
\hline $5 a$ & 17.6 & 15.3 \\
\hline $5 b$ & 18.6 & 16.0 \\
\hline $5 c$ & 15.6 & 15.0 \\
\hline $5 d$ & 16.3 & 15.6 \\
\hline $5 e$ & 15.0 & 16.6 \\
\hline $5 f$ & 16.6 & 16.6 \\
\hline $5 g$ & 18.0 & 16.0 \\
\hline $5 \mathrm{~h}$ & 20.0 & 21.0 \\
\hline $6 a$ & 18.6 & 19.3 \\
\hline $6 b$ & 18.6 & 19.3 \\
\hline $6 c$ & 14.0 & 15.3 \\
\hline $6 d$ & 16.6 & 17.3 \\
\hline $6 e$ & 14.6 & 13.0 \\
\hline $6 f$ & 13.6 & 14.3 \\
\hline $6 g$ & 13.6 & 14.6 \\
\hline $6 \mathrm{~h}$ & 19.0 & 18.0 \\
\hline Ciprofloxacin & 26.0 & 24.0 \\
\hline
\end{tabular}

avalues including diameter of the well $(8 \mathrm{~mm})$ are means of three replicates
In case of Gram-positive bacteria, compounds $\mathbf{4 h}, \mathbf{5 b}$, $5 h, 6 a, 6 b$, and $6 h$ were found to be most effective against $S$. aureus with zone of inhibition ranging between $18.6 \mathrm{~mm}$ and $20.0 \mathrm{~mm}$ and the compounds $\mathbf{5 h}$, $\mathbf{6 a}$, and $\mathbf{6 b}$ were most effective against $B$. subtillis with zone of inhibition ranging between $19.3 \mathrm{~mm}$ and 21.0 $\mathrm{mm}$ (Table 3).

In whole series, compounds $\mathbf{4 a}, \mathbf{4 h}$, and $\mathbf{5 h}$ showed maximum antibacterial activity against $S$. aureus (MIC $64 \mu \mathrm{g} / \mathrm{mL}$ ) and compounds 5 h (MIC $32 \mu \mathrm{g} / \mathrm{mL}$ ), $6 \mathbf{a}$ \&6h (MIC $64 \mu \mathrm{g} / \mathrm{mL}$ ) against B. subtillis (Table 4).

\section{Conclusions}

We have described herein an efficient and convenient synthesis of three series of pyrazolyl-2, 4-TZDs (4-6) by Knoevenagel condensation. All the 24 compounds synthesized were characterized by spectral and elemental analytical data and evaluated for their in vitro antifungal and antibacterial activities. Results of the antifungal activity were found to be comparable with the reference compound. On the other hand, antibacterial activity was best observed for Gram-positive bacteria only, none of the compounds showed activity against Gram-negative bacteria.

\section{Table 4 MIC of the compounds 4-6}

\begin{tabular}{|c|c|c|}
\hline \multirow[t]{2}{*}{ Compounds } & \multicolumn{2}{|c|}{ MIC $(\mu \mathrm{g} / \mathrm{mL})$} \\
\hline & S. aureus & B. subtilis \\
\hline $4 a$ & 128 & 128 \\
\hline $4 b$ & 128 & 128 \\
\hline $4 c$ & 128 & 128 \\
\hline $4 d$ & 128 & 128 \\
\hline $4 e$ & 128 & 128 \\
\hline $4 f$ & 128 & 128 \\
\hline $4 g$ & 128 & 128 \\
\hline $4 h$ & 64 & 128 \\
\hline $5 a$ & 128 & 128 \\
\hline $5 b$ & 128 & 128 \\
\hline $5 c$ & 128 & 128 \\
\hline $5 d$ & 128 & 128 \\
\hline $5 e$ & 128 & 128 \\
\hline $5 f$ & 128 & 128 \\
\hline $5 g$ & 64 & 128 \\
\hline $5 \mathrm{~h}$ & 64 & 32 \\
\hline $6 a$ & 64 & 64 \\
\hline $6 b$ & 128 & 64 \\
\hline $6 c$ & 128 & 128 \\
\hline $6 d$ & 128 & 128 \\
\hline $6 e$ & 128 & 128 \\
\hline $6 f$ & 128 & 128 \\
\hline $6 g$ & 128 & 128 \\
\hline $6 \mathrm{~h}$ & 64 & 64 \\
\hline Ciprofloxacin & 5 & 5 \\
\hline
\end{tabular}




\section{Experimental}

\subsection{General remarks}

Melting points (mps) were taken on slides in an electrical apparatus Labindia visual melting range apparatus and are uncorrected. Calibration of melting point apparatus was done using benzoic acid as reference. IR spectra were recorded on a Perkin-Elmer 1800 FT-IR spectrophotometer. ${ }^{1} \mathrm{H}$ NMR spectra (see additional files $1,2,3,4,5,6,7,8,9,10,11,12,13,14,15,16,17,18$, $19,20,21,22,23,24)$ were recorded on a Bruker $300 \&$ $400 \mathrm{MHz}$ instrument using tetramethylsilane as an internal standard. Mass spectra were recorded on 2500 eV (ESI Source) using a water's Q-TOF microinstrument and elemental analysis on Perkin-Elmer 2400 instrument. All the reagents were purchased from the commercial sources and were used without further purification.

\subsection{Preparation of ethyl 2-((Z)-5-((3-aryl-1-phenyl-1H- pyrazol-4-yl)methylene)-2, 4-dioxothiazolidin-3-yl)acetates (4a-h)}

Typical procedure: A mixture of 1, 3-diphenyl- $1 H$-pyrazol-4-carboxaldehyde 1a $(0.5 \mathrm{~g}, 2 \mathrm{mmol})$ and ethyl 2-(2, 4-dioxothiazolidin-3-yl)acetate 3 ( $0.4 \mathrm{~g}, 2 \mathrm{mmol})$ in ethanol $(20 \mathrm{~mL})$ and $2-3$ drops of piperidine was refluxed for 4-5 h. A solid was separated out of the reaction mixture within $15-20 \mathrm{~min}$ and the refluxing was continued for 4-5 $\mathrm{h}$ to complete the reaction. The reaction mixture was cooled to room temperature, filtered, and washed with ethanol to give the pure product $4 a(0.87 \mathrm{~g}, 90 \%$ yield $)$.

The other derivatives $\mathbf{4 b} \mathbf{b} \mathbf{h}$ were synthesized by adopting the similar procedure.

\subsection{Ethyl 2-((Z)-2, 4-dioxo-5-((1, 3-diphenyl-1H-pyrazol-4-} yl)methylene)thiazolidin-3-yl)acetate (4a)

IR $\left(v_{\max }, \mathrm{KBr}\right) \mathrm{cm}^{-1}: 1736,1690,1612,1535,1504,1450$, 1373, 1311, 1227, 1142, 1103, 1065, 1026. ${ }^{1} \mathrm{H}$ NMR $\left(\mathrm{CDCl}_{3}, 400 \mathrm{MHz}, \delta\right): 8.213$ (s, 1H, Pyrazolyl H), 7.963 $(\mathrm{s}, 1 \mathrm{H},=\mathrm{CH}), 7.817-7.795(\mathrm{~m}, 2 \mathrm{H}, \mathrm{Ar} \mathrm{H}), 7.678-7.654$ (m, 2H, Ar H), 7.549-7.471 (m, 5H, Ar H), 7.414-7.377 (m, 1H, Ar H), 4.473 (s, 2H, $\mathrm{NCH}_{2}$ ), 4.275-4.222 (q, 2H, $-\mathrm{OCH}_{2} \mathrm{CH}_{3}$ ), 1.319-1.283 (t, 3H, $-\mathrm{OCH}_{2} \mathrm{CH}_{3}$ ). MS (ESI+) $m / z$ 434 $[\mathrm{M}+\mathrm{H}]$. Anal. Found: C, 63.3; H, 4.6; N, 9.5. $\mathrm{C}_{23} \mathrm{H}_{19} \mathrm{~N}_{3} \mathrm{O}_{4} \mathrm{~S}$ requires $\mathrm{C}, 63.73 ; \mathrm{H}, 4.42 ; \mathrm{N}, 9.69 \%$.

4.4. Ethyl 2-((Z)-2, 4-dioxo-5-((1-phenyl-3-p-tolyl-1Hpyrazol-4-yl)methylene)thiazolidin-3-yl)acetate (4b) IR $\left(v_{\max }, \mathrm{KBr}\right) \mathrm{cm}^{-1}: 1736,1690,1605,1520,1450,1373$, 1311, 1219, 1142, 1095, 1026. ${ }^{1} \mathrm{H}$ NMR (DMSO-d 6,400 $\mathrm{MHz}, \delta): 8.812$ (s, 1H, Pyrazolyl H), 8.041-8.022 (m, 2H, Ar H), 7.739 (s, 1H, =CH) 7.598-7.536 (m, 4H, Ar H), 7.448-7.379 (m, 3H, Ar H), 4.480 (s, $2 \mathrm{H}, \mathrm{NCH}_{2}$ ), 4.1994.145 (q, $2 \mathrm{H},-\mathrm{OCH}_{2} \mathrm{CH}_{3}$ ), 2.405 (s, 3H, $\mathrm{Ph} \mathrm{CH}_{3}$ ), 1.231- $1.195\left(\mathrm{t}, 3 \mathrm{H},-\mathrm{OCH}_{2} \mathrm{CH}_{3}\right)$. MS (ESI+) $m / z 448[\mathrm{M}+\mathrm{H}]$. Anal. Found: C, 64.0; H, 4.98; N, 9.2. $\mathrm{C}_{24} \mathrm{H}_{21} \mathrm{~N}_{3} \mathrm{O}_{4} \mathrm{~S}$ requires $\mathrm{C}, 64.41 ; \mathrm{H}, 4.73, \mathrm{~N}, 9.39 \%$.

4.5. Ethyl 2-((Z)-5-((3-(4-methoxyphenyl)-1-phenyl-1Hpyrazol-4-yl)methylene)-2, 4-dioxothiazolidin-3-yl)acetate (4c)

IR $\left(v_{\max }, \mathrm{KBr}\right) \mathrm{cm}^{-1}: 1736,1690,1612,1520,1450,1373$, $1311,1296,1227,1180,1142,1095,1026,1018 .{ }^{1} \mathrm{H}$ NMR (TFA-d $1,400 \mathrm{MHz}, \delta): 8.483$ (s, 1H, Pyrazolyl H), $7.917(\mathrm{~s}, 1 \mathrm{H},=\mathrm{CH}), 7.667-7.583(\mathrm{~m}, 7 \mathrm{H}, \mathrm{Ar} \mathrm{H}), 7.179$ 7.157 (d, $2 \mathrm{H}, \mathrm{Ar} \mathrm{H}, J=8.8 \mathrm{~Hz}), 4.620\left(\mathrm{~s}, 2 \mathrm{H}, \mathrm{NCH}_{2}\right)$, 4.345-4.291 (q, $2 \mathrm{H}, \mathrm{CH}_{2} \mathrm{CH}_{3}$ ), 3.922 (s, 3H, Ph $\mathrm{OCH}_{3}$ ), 1.304-1.269 (t, 3H, $\mathrm{CH}_{3} \mathrm{CH}_{2}$ ). MS (ESI+) $\mathrm{m} / z$ 464 [M $+\mathrm{H}]$. Anal. Found: C, 61.8; H, 4.1; N, 8.6. $\mathrm{C}_{24} \mathrm{H}_{21} \mathrm{~N}_{3} \mathrm{O}_{5} \mathrm{~S}$ requires $C$, 62.19; $\mathrm{H}, 4.57 ; \mathrm{N}, 9.07 \%$.

4.6. Ethyl 2-((Z)-5-((3-(4-chlorophenyl)-1-phenyl-1Hpyrazol-4-yl)methylene)-2, 4-dioxothiazolidin-3-yl)acetate (4d)

IR $\left(v_{\max }, \mathrm{KBr}\right) \mathrm{cm}^{-1}: 1736,1690,1612,1528,1443$, 1373, 1311, 1227, 1142, 1095, 1011. ${ }^{1} \mathrm{H}$ NMR $\left(\right.$ TFA-d $_{1}$, $400 \mathrm{MHz}, \delta$ ): 8.657 (s, 1H, Pyrazolyl H), 8.052 (s, 1H, $=\mathrm{CH}), 7.832-7.748(\mathrm{~m}, 5 \mathrm{H}, \mathrm{Ar} \mathrm{H}), 7.748-7.724(\mathrm{~m}, 4 \mathrm{H}$, Ar $\mathrm{H}), 4.789\left(\mathrm{~s}, 2 \mathrm{H}, \mathrm{NCH}_{2}\right), 4.515-4.462(\mathrm{q}, 2 \mathrm{H}$, $-\mathrm{OCH}_{2} \mathrm{CH}_{3}$ ), 1.476-1.440 (t, $3 \mathrm{H},-\mathrm{OCH}_{2} \mathrm{CH}_{3}$ ). MS (ESI +) $m / z 454[\mathrm{M}+\mathrm{H}]$. Anal. Found: C, 58.6; H, 3.9; N, 8.7. $\mathrm{C}_{23} \mathrm{H}_{18} \mathrm{ClN}_{3} \mathrm{O}_{4} \mathrm{~S}$ requires $\mathrm{C}, 59.04 ; \mathrm{H}, 3.88 ; \mathrm{N}$, $8.98 \%$.

\subsection{Ethyl 2-((Z)-5-((3-(4-fluorophenyl)-1-phenyl-1H-} pyrazol-4-yl)methylene)-2, 4-dioxothiazolidin-3-yl)acetate (4e)

IR $\left(v_{\max }, \mathrm{KBr}\right) \mathrm{cm}^{-1}: 1736,1697,1612,1512,1450,1373$, 1311, 1234, 1142, 1095, 1026. ${ }^{1} \mathrm{H}$ NMR $\left(\right.$ TFA-d $_{1}, 400$ $\mathrm{MHz}, \delta): 8.489$ (s, 1H, Pyrazolyl H), 7.884 (s, 1H, =CH), 7.652-7.584 (m, 7H, Ar H), 7.290-7.247 (m, 2H, Ar H), 4.624 (s, 2H, $\mathrm{NCH}_{2}$ ), 4.351-4.297 (q, 2H, $-\mathrm{OCH}_{2} \mathrm{CH}_{3}$ ), 1.311-1.275 (t, 3H, $-\mathrm{OCH}_{2} \mathrm{CH}_{3}$ ). MS (ESI+) $\mathrm{m} / z 437$ [M $+\mathrm{H}$ ]. Anal. Found: C, 61.0; H, 4.2; N, 9.2. $\mathrm{C}_{23} \mathrm{H}_{18} \mathrm{FN}_{3} \mathrm{O}_{4} \mathrm{~S}$ requires $C, 61.19 ; \mathrm{H}, 4.02 ; \mathrm{N}, 9.31 \%$.

4.8. Ethyl 2-((Z)-5-((3-(4-bromophenyl)-1-phenyl-1Hpyrazol-4-yl)methylene)-2, 4-dioxothiazolidin-3-yl)acetate (4f)

IR $\left(v_{\max }, \mathrm{KBr}\right) \mathrm{cm}^{-1}: 1736,1690,1605,1528,1443,1373$, 1311, 1227, 1142, 1095, 1003. ${ }^{1} \mathrm{H}$ NMR $\left(\right.$ TFA-d $_{1}, 400$ $\mathrm{MHz}, \delta): 8.488$ (s, 1H, Pyrazolyl H), 7.896 (s, 1H, =CH), 7.750-7.729 (m, 2H, Ar H), 7.650-7.588 (m, 5H, Ar H), 7.489-7.467 (d, 2H, Ar H,J = 8.8 Hz) $4.633(\mathrm{~s}, 2 \mathrm{H}$, $\mathrm{NCH}_{2}$ ), 4.359-4.305 (q, 2H, $\left.-\mathrm{OCH}_{2} \mathrm{CH}_{3}\right), 1.319-1.283$ (t, $\left.3 \mathrm{H},-\mathrm{OCH}_{2} \mathrm{CH}_{3}\right)$. MS (ESI+) $m / z 497[\mathrm{M}+\mathrm{H}]$. Anal. Found: $\mathrm{C}, 53.7 ; \mathrm{H}, 3.4 ; \mathrm{N}, 8.0 . \mathrm{C}_{23} \mathrm{H}_{18} \mathrm{BrN}_{3} \mathrm{O}_{4} \mathrm{~S}$ requires C, $53.91 ; \mathrm{H}, 3.54 ; \mathrm{N}, 8.20 \%$. 
4.9. Ethyl 2-((Z)-5-((3-(4-hydroxyphenyl)-1-phenyl-1Hpyrazol-4-yl)methylene)-2, 4-dioxothiazolidin-3-yl)acetate (4g)

IR $\left(v_{\max }, \mathrm{KBr}\right) \mathrm{cm}^{-1}: 3387,1736,1682,1605,1520,1373$, 1319, 1234, 1142, 1103, 1026. ${ }^{1} \mathrm{H}$ NMR (DMSO-d 6,400 $\mathrm{MHz}, \delta): 9.850$ (bs, 1H, OH), 8.773 (s, 1H, Pyrazolyl H), 8.027-8.007 (m, 2H, Ar H), 7.734 (s, 1H, =CH), 7.5887.549 (m, 2H, Ar H), 7.474-7.452 (d, 2H, Ar H, $J=8.8$ $\mathrm{Hz}$ ), 7.435-7.398 (m, 1H, Ar H), 6.955-6.933 (d, 2H, Ar $\mathrm{H}, J=8.8 \mathrm{~Hz}$ ), 4.479 (s, 2H, $\mathrm{NCH}_{2}$ ), 4.199-4.146 (q, 2H, $-\mathrm{OCH}_{2} \mathrm{CH}_{3}$ ), 1.232-1.196 (t, 3H, $-\mathrm{OCH}_{2} \mathrm{CH}_{3}$ ). MS (ESI+) $m / z 435[\mathrm{M}+\mathrm{H}]$. Anal. Found: C, 61.3; H, 4.4; N, 9.1. $\mathrm{C}_{23} \mathrm{H}_{19} \mathrm{~N}_{3} \mathrm{O}_{5} \mathrm{~S}$ requires $\mathrm{C}, 61.46 ; \mathrm{H}, 4.26 ; \mathrm{N}, 9.35 \%$.

4.10. Ethyl 2-((Z)-5-((3-(4-nitrophenyl)-1-phenyl-1Hpyrazol-4-yl)methylene)-2, 4-dioxothiazolidin-3-yl)acetate (4h)

IR $\left(v_{\max }, \mathrm{KBr}\right) \mathrm{cm}^{-1}: 1736,1697,1620,1528,1350,1319$, 1234, 1142, 1095. ${ }^{1} \mathrm{H}$ NMR (TFA-d $1,400 \mathrm{MHz}, \delta$ ): 8.482-8.460 (d, 2H, Ar H, $J=8.8$ Hz), 8.391 (s, 1H, Pyrazolyl H), 7.957 (s, 1H, =CH), 7.895-7.874 (d, 2H, Ar H, $J=8.4 \mathrm{~Hz}), 7.664-7.652(\mathrm{~m}, 2 \mathrm{H}$, Ar H), 7.586-7.573 (m, $3 \mathrm{H}, \mathrm{Ar} \mathrm{H}), 4.666$ (s, 2H, $\mathrm{NCH}_{2}$ ), 4.388-4.334 (q, 2H, $-\mathrm{OCH}_{2} \mathrm{CH}_{3}$ ), 1.347-1.311 (t, 3H, $-\mathrm{OCH}_{2} \mathrm{CH}_{3}$ ). MS (ESI+) $m / z 465[\mathrm{M}+\mathrm{H}]$. Anal. Found: C, 57.4; H, 3.9; N, 11.6. $\mathrm{C}_{23} \mathrm{H}_{18} \mathrm{~N}_{4} \mathrm{O}_{6} \mathrm{~S}$ requires $\mathrm{C}, 57.73 ; \mathrm{H}, 3.79 ; \mathrm{N}, 11.71 \%$.

4.11. Preparation of methyl 2-((Z)-5-((3-aryl-1-phenyl-1Hpyrazol-4-yl)methylene)-2, 4-dioxothiazolidin-3-yl)acetates (5a-h)

Typical procedure: A mixture of 1, 3-diphenyl-1H-pyrazol-4-carboxaldehyde 1a $(0.5 \mathrm{~g}, 2 \mathrm{mmol})$ and methyl 2(2, 4-dioxothiazolidin-3-yl)acetate $2(0.38 \mathrm{~g}, 2 \mathrm{mmol})$ in methanol $(20 \mathrm{ml})$ and $2-3$ drops of piperidine was refluxed 4-5 h. A solid was separated out of the reaction mixture within 15-20 min and the refluxing was continued for 4-5 h to complete the reaction. The reaction mixture was cooled to room temperature, filtered and washed with methanol to give the pure product $5 \mathbf{a}(0.84$ g, $92 \%$ yield).

The other derivatives $\mathbf{5 b} \mathbf{b} \mathbf{h}$ were synthesized by adopting the similar procedure.

4.12. Methyl 2-((Z)-2, 4-dioxo-5-((1, 3-diphenyl-1H-pyrazol4-yl)methylene)thiazolidin-3-yl)acetate (5a)

IR $\left(v_{\max }, \mathrm{KBr}\right) \mathrm{cm}^{-1}: 1744,1690,1605,1535,1443$, 1366, 1311, 1234, 1142, 1103, 1011. ${ }^{1} \mathrm{H}$ NMR (DMSO$\left.\mathrm{d}_{6}, 400 \mathrm{MHz}, \delta\right): 8.828(\mathrm{~s}, 1 \mathrm{H}$, Pyrazolyl H), 8.069$8.029(\mathrm{~m}, 2 \mathrm{H}$, Ar H) $7.745(\mathrm{~s}, 1 \mathrm{H},=\mathrm{CH}), 7.685-7.649$ (m, 2H, Ar H), 7.601-7.537 (m, 5H, Ar H), 7.453-7.417 $(\mathrm{m}, 1 \mathrm{H}, \operatorname{Ar~H}), 4.501\left(\mathrm{~s}, 2 \mathrm{H}, \mathrm{NCH}_{2}\right), 3.711(\mathrm{~s}, 3 \mathrm{H}$, $\left.\mathrm{COOCH}_{3}\right)$. MS (ESI+) $m / z 406[\mathrm{M}+\mathrm{H}]$. Anal. Found: C, 62.7; $\mathrm{H}, 4.2 ; \mathrm{N}, 9.9 . \mathrm{C}_{22} \mathrm{H}_{17} \mathrm{~N}_{3} \mathrm{O}_{4} \mathrm{~S}$ requires $\mathrm{C}, 63.00$; H, 4.09; N, 10.02\%.
4.13. Methyl 2-((Z)-2, 4-dioxo-5-((1-phenyl-3-p-tolyl-1Hpyrazol-4-yl)methylene)thiazolidin-3-yl)acetate (5b) IR $\left(v_{\max }, \mathrm{KBr}\right) \mathrm{cm}^{-1}: 1744,1690,1605,1512,1443,1366$, 1319, 1234, 1142, 1103, 1011. ${ }^{1} \mathrm{H}$ NMR $\left(\right.$ TFA-d $_{1}, 400$ $\mathrm{MHz}, \delta): 8.501$ (s, 1H, Pyrazolyl H), $7.924(\mathrm{~s}, 1 \mathrm{H},=\mathrm{CH})$, 7.626 (m, 5H, Ar H), 7.492-7.472 (m, 2H, Ar H), 7.417$7.398(\mathrm{~m}, 2 \mathrm{H}, \mathrm{Ar} \mathrm{H}), 4.632$ (s, 2H, $\left.\mathrm{NCH}_{2}\right), 3.711(\mathrm{~s}, 3 \mathrm{H}$, $\mathrm{COOCH}_{3}$ ), 2.404 (s, 3H, Ph $\mathrm{CH}_{3}$ ). MS (ESI+) $m / z 419$ $[\mathrm{M}+\mathrm{H}]$. Anal. Found: C, 63.6; H, 4.5; N, 9.4. $\mathrm{C}_{23} \mathrm{H}_{19} \mathrm{~N}_{3} \mathrm{O}_{4} \mathrm{~S}$ requires $\mathrm{C}, 63.73 ; \mathrm{H}, 4.42 ; \mathrm{N}, 9.69 \%$.

4.14. Methyl 2-((Z)-5-((3-(4-methoxyphenyl)-1-phenyl-1Hpyrazol-4-yl)methylene)-2, 4-dioxothiazolidin-3-yl)acetate (5c)

IR $\left(v_{\max }, \mathrm{KBr}\right) \mathrm{cm}^{-1}: 1744,1690,1612,1520,1443,1366$, 1296, 1242, 1180, 1142, 1103, 1018. ${ }^{1} \mathrm{H}$ NMR (TFA-d ${ }_{1}$, $400 \mathrm{MHz}, \delta): 8.477$ (s, 1H, Pyrazolyl H), 7.915 (s, 1H, $=\mathrm{CH}), 7.665-7.568(\mathrm{~m}, 6 \mathrm{H}, \mathrm{Ar} \mathrm{H}), 7.178-7.156(\mathrm{~d}, 2 \mathrm{H}$, Ar $\mathrm{H}, J=8.8 \mathrm{~Hz}), 4.630\left(\mathrm{~s}, 2 \mathrm{H}, \mathrm{NCH}_{2}\right), 3.923(\mathrm{~s}, 3 \mathrm{H}$, $\mathrm{COOCH}_{3}$ ), 3.859 (s, 3H, Ph $\mathrm{OCH}_{3}$ ). MS (ESI+) $\mathrm{m} / z 436$ $[\mathrm{M}+\mathrm{H}]$. Anal. Found: C, 61.3; $\mathrm{H}, 4.4 ; \mathrm{N}, 9.2$. $\mathrm{C}_{23} \mathrm{H}_{19} \mathrm{~N}_{3} \mathrm{O}_{5} \mathrm{~S}$ requires $\mathrm{C}, 61.46 ; \mathrm{H}, 4.26 ; \mathrm{N}, 9.35 \%$.

\subsection{Methyl 2-((Z)-5-((3-(4-chlorophenyl)-1-phenyl-1H-} pyrazol-4-yl)methylene)-2, 4-dioxothiazolidin-3-yl)acetate (5d)

IR $\left(v_{\max }, \mathrm{KBr}\right) \mathrm{cm}^{-1}: 1744,1697,1605,1528,1443,1366$, 1319, 1242, 1142, 1103, 1011. ${ }^{1} \mathrm{H}$ NMR $($ TFA-d 1,400 $\mathrm{MHz}, \delta): 8.476$ (s, 1H, Pyrazolyl H), $7.884(\mathrm{~s}, 1 \mathrm{H},=\mathrm{CH})$, 7.618-7.552 (m, 9H, Ar H), 4.630 (s, 2H, $\mathrm{NCH}_{2}$ ), 3.861 $\left(\mathrm{s}, 3 \mathrm{H}, \mathrm{COOCH}_{3}\right)$. MS (ESI+) $m / z 440[\mathrm{M}+\mathrm{H}]$. Anal. Found: $\mathrm{C}, 58.0 ; \mathrm{H}, 3.6 ; \mathrm{N}, 9.1 . \mathrm{C}_{22} \mathrm{H}_{16} \mathrm{~N}_{3} \mathrm{O}_{4} \mathrm{~S}$ requires $\mathrm{C}$, $58.21 ; \mathrm{H}, 3.55 ; \mathrm{N}, 9.26 \%$.

Methyl 2-((Z)-5-((3-(4-fluorophenyl)-1-phenyl-1H-pyrazol-4yl)methylene)-2, 4-dioxothiazolidin-3-yl)acetate (5e) IR $\left(v_{\max }, \mathrm{KBr}\right) \mathrm{cm}^{-1}: 1744,1697,1612,1520,1404,1366$, 1319, 1234, 1149, 1095. ${ }^{1} \mathrm{H}$ NMR (TFA-d $\left.1,400 \mathrm{MHz}, \delta\right)$ : 8.494 (s, 1H, Pyrazolyl H), 7.893 (s, 1H, =CH), 7.6507.616 (m, 7H, Ar H), 7.300-7.258 (m, 2H, Ar H), 4.663 $\left(\mathrm{s}, 2 \mathrm{H}, \mathrm{NCH}_{2}\right), 3.876\left(\mathrm{~s}, 3 \mathrm{H}, \mathrm{COOCH}_{3}\right) . \mathrm{MS}(\mathrm{ESI}+) \mathrm{m} / z$ $424[\mathrm{M}+\mathrm{H}]$. Anal. Found: $\mathrm{C}, 60.2 ; \mathrm{H}, 3.8 ; \mathrm{N}, 9.5$. $\mathrm{C}_{22} \mathrm{H}_{16} \mathrm{FN}_{3} \mathrm{O}_{4} \mathrm{~S}$ requires $\mathrm{C}, 60.40 ; \mathrm{H}, 3.69 ; \mathrm{N}, 9.61 \%$.

Methyl 2-((Z)-5-((3-(4-bromophenyl)-1-phenyl-1H-pyrazol4-yl)methylene)-2, 4-dioxothiazolidin-3-yl)acetate (5f) IR $\left(v_{\max }, \mathrm{KBr}\right) \mathrm{cm}^{-1}: 1744,1697,1612,1520,1404,1366$, 1319, 1234, 1149, 1095. ${ }^{1} \mathrm{H}$ NMR $\left(\mathrm{CDCl}_{3}+\right.$ TFA-d $_{1}, 400$ $\mathrm{MHz}, \delta): 8.250$ (s, 1H, Pyrazolyl H), 7.899 (s, 1H, =CH), 7.750-7.730 (d, 2H, Ar H, $J=8.0 \mathrm{~Hz}), 7.660-7.611(\mathrm{~m}$, $5 \mathrm{H}$, Ar H), 7.500-7.480 (d, 2H, Ar H, J = 8.00 Hz), 4.652 (s, $2 \mathrm{H}, \mathrm{NCH}_{2}$ ), 3.901 (s, 3H, $\mathrm{COOCH}_{3}$ ). MS (ESI+) $\mathrm{m} / z$ $483[\mathrm{M}+\mathrm{H}]$. Anal. Found: $\mathrm{C}, 52.9 ; \mathrm{H}, 3.4 ; \mathrm{N}, 8.2$. $\mathrm{C}_{22} \mathrm{H}_{16} \mathrm{BrN}_{3} \mathrm{O}_{4} \mathrm{~S}$ requires $\mathrm{C}, 53.02 ; \mathrm{H}, 3.24 ; \mathrm{N}, 8.43 \%$. 
Methyl 2-((Z)-5-((3-(4-hydroxyphenyl)-1-phenyl-1H-pyrazol4-yl)methylene)-2, 4-dioxothiazolidin-3-yl)acetate (5g) IR $\left(v_{\max }, \mathrm{KBr}\right) \mathrm{cm}^{-1}: 3348,1736,1682,1605,1512,1443$, $1412,1373,1311,1234,1211,1142,1103 .{ }^{1} \mathrm{H}$ NMR (DMSO-d $6,400 \mathrm{MHz}, \delta$ ): 9.863 (s, 1H, Ph OH), 8.764 (s, 1H, Pyrazolyl H), 8.023-8.003 (m, 2H, Ar H), 7.730 (s, $1 \mathrm{H},=\mathrm{CH}), 7.585-7.546(\mathrm{~m}, 2 \mathrm{H}, \operatorname{Ar} \mathrm{H}), 7.471-7.450(\mathrm{~d}$, $2 \mathrm{H}$, Ar $\mathrm{H}, J=8.4 \mathrm{~Hz}), 7.434-7.395(\mathrm{~m}, 1 \mathrm{H}$, Ar $\mathrm{H})$, 6.954-6.933 (d, 2H, Ar $\mathrm{H}, J=8.4 \mathrm{~Hz}), 4.499(\mathrm{~s}, 2 \mathrm{H}$, $\mathrm{NCH}_{2}$ ), $3.712\left(\mathrm{~s}, 3 \mathrm{H}, \mathrm{COOCH}_{3}\right)$. MS (ESI+) $\mathrm{m} / z 450[\mathrm{M}$ $+\mathrm{H}$ ]. Anal. Found: C, 60.5; H, 4.0; N, 9.5. $\mathrm{C}_{22} \mathrm{H}_{17} \mathrm{~N}_{3} \mathrm{O}_{5} \mathrm{~S}$ requires $C, 60.68 ; H, 3.93 ; \mathrm{N}, 9.65 \%$.

Methyl 2-((Z)-5-((3-(4-nitrophenyl)-1-phenyl-1H-pyrazol-4yl)methylene)-2, 4-dioxothiazolidin-3-yl)acetate (5h) IR $\left(v_{\max }, \mathrm{KBr}\right) \mathrm{cm}^{-1}: 1744,1690,1605,1528,1412,1342$, 1273, 1219, 1142, 1103. ${ }^{1} \mathrm{H}$ NMR $\left(\mathrm{CDCl}_{3}+\mathrm{TFA}_{-} \mathrm{d}_{1}, 400\right.$ $\mathrm{MHz}, \delta): 8.454-8.434(\mathrm{~d}, 2 \mathrm{H}, \mathrm{Ar} \mathrm{H}, J=8.8 \mathrm{~Hz}), 8.261$ 8.247 (m, 2H, Ar H), 7.906-7.834 (m, 3H, Ar H), 7.7107.689 (m, 2H, Ar H), 7.637-7.571 (m, 2H, Ar H), 4.642 $\left(\mathrm{s}, 2 \mathrm{H}, \mathrm{NCH}_{2}\right), 3.985\left(\mathrm{~s}, 3 \mathrm{H}, \mathrm{COOCH}_{3}\right) . \mathrm{MS}(\mathrm{ESI}+) \mathrm{m} / z$ $450[\mathrm{M}+\mathrm{H}]$. Anal. Found: C, 58.7; H, 3.6; N, 11.8 . $\mathrm{C}_{22} \mathrm{H}_{16} \mathrm{~N}_{4} \mathrm{O}_{6} \mathrm{~S}$ requires $\mathrm{C}, 58.89 ; \mathrm{H}, 3.47 ; \mathrm{N}, 12.06 \%$.

Preparation of 2-((Z)-5-((3-aryl-1-phenyl-1H-pyrazol-4-yl) methylene)-2, 4-dioxothiazolidin-3-yl)acetic acid (6a-h) Typical procedure: A mixture of ethyl 2-((Z)-2, 4-dioxo5-((1, 3-diphenyl-1H-pyrazol-4-yl)methylene)thiazolidin3 -yl)acetate $4 \mathbf{a}(0.5 \mathrm{~g}, 1.1 \mathrm{mmol}), 10 \mathrm{~mL}$ of $50 \%$ aqueous sulphuric acid in $35 \mathrm{~mL}$ acetic acid was refluxed for 5-6 h. On cooling, the reaction mixture was poured onto crushed ice. Solid separated was filtered, washed with excess of cold water followed by alcohol to obtain white solid 6a $(0.47 \mathrm{~g}, 94 \%)$. Similarly, 6a can also be obtained from 5 a by hydrolysis.

All other derivatives $\mathbf{6 b}-\mathbf{h}$ were synthesized by adopting the similar procedure.

\section{2-((Z)-2, 4-Dioxo-5-((1, 3-diphenyl-1H-pyrazol-4-yl)} methylene)thiazolidin-3-yl)acetic acid (6a)

IR $\left(v_{\max }, \mathrm{KBr}\right) \mathrm{cm}^{-1}: 3472,3418,1744,1697,1605,1528$, 1504, 1443, 1373, 1319, 1219, 1149, 1103, 1102, 1057, 1003. ${ }^{1} \mathrm{H}$ NMR (DMSO-d $6,300 \mathrm{MHz}, \delta$ ): 8.807 (s, $1 \mathrm{H}$, Pyrazolyl H), 8.040-8.018 (m, 2H, Ar H), 7.729-7.434 $(\mathrm{m}, 9 \mathrm{H}, \mathrm{ArH}+=\mathrm{CH}), 4.359\left(\mathrm{~s}, 2 \mathrm{H}, \mathrm{NCH}_{2}\right) . \mathrm{MS}(\mathrm{ESI}+)$ $m / z 392[\mathrm{M}+\mathrm{H}]$. Anal. Found: $\mathrm{C}, 62.1 ; \mathrm{H}, 3.8 ; \mathrm{N}, 10.2$. $\mathrm{C}_{21} \mathrm{H}_{15} \mathrm{~N}_{3} \mathrm{O}_{4} \mathrm{~S}$ requires $\mathrm{C}, 62.21 ; \mathrm{H}, 3.73 ; \mathrm{N}, 10.36 \%$.

\section{2-((Z)-2, 4-Dioxo-5-((1-phenyl-3-p-tolyl-1H-pyrazol-4-yl)} methylene)thiazolidin-3-yl)acetic acid (6b)

IR $\left(v_{\max }, \mathrm{KBr}\right) \mathrm{cm}^{-1}: 1744,1697,1605,1512,1450,1389$, 1319, 1227, 1149, 1103, 1003. ${ }^{1} \mathrm{H}$ NMR (DMSO-d 6,300 $\mathrm{MHz}, \delta): 8.795$ (s, 1H, Pyrazolyl H), 8.045-8.015 (m, 2H, Ar H), 7.727 (s, $1 \mathrm{H},=\mathrm{CH})$, 7.603-7.530 (m, 4H, Ar H),
7.451-7.373 (m, 3H, Ar H), 4.366 (s, 2H, $\mathrm{NCH}_{2}$ ), 2.405 (s, 3H, $\mathrm{CH}_{3}$ ). MS (ESI+) $m / z 406[\mathrm{M}+\mathrm{H}]$. Anal. Found: C, 62.8; $\mathrm{H}, 4.2 ; \mathrm{N}, 9.9 . \mathrm{C}_{22} \mathrm{H}_{17} \mathrm{~N}_{3} \mathrm{O}_{4} \mathrm{~S}$ requires $\mathrm{C}$, 63.00; H, 4.09; N, $10.02 \%$.

\section{2-((Z)-5-((3-(4-Methoxyphenyl)-1-phenyl-1H-pyrazol-4-yl)} methylene)-2, 4-dioxothiazolidin-3-yl)acetic acid (6c) IR $\left(v_{\max }, \mathrm{KBr}\right) \mathrm{cm}^{-1}: 1736,1690,1612,1520,1450,1396$, 1296, 1242, 1180, 1142, 1103, 1018. ${ }^{1} \mathrm{H}$ NMR (DMSO-d ${ }_{6}$, $300 \mathrm{MHz}, \delta$ ): 8.782 (s, 1H, Pyrazolyl H), 8.037-8.011 (m, 2H, Ar H), 7.722 (s, 1H, =CH), 7.599-7.548 (m, 4H, Ar H), 7.447-7.398 (m, 1H, Ar H), 7.149-7.120 (d, 2H, Ar H, $J=$ $8.7 \mathrm{~Hz}), 4.365$ (s, 2H, $\mathrm{NCH}_{2}$ ), 3.842 (s, 3H, $\left.\mathrm{OCH}_{3}\right) . \mathrm{MS}$ (ESI+) $m / z 422[\mathrm{M}+\mathrm{H}]$. Anal. Found: C, 60.5; H, 3.8, N, 14.20. $\mathrm{C}_{22} \mathrm{H}_{17} \mathrm{~N}_{3} \mathrm{O}_{5} \mathrm{~S}$ requires $\mathrm{C}, 60.68 ; \mathrm{H}, 3.93 ; \mathrm{N}, 9.65 \%$.

\section{2-((Z)-5-((3-(4-Chlorophenyl)-1-phenyl-1H-pyrazol-4-yl)} methylene)-2, 4-dioxothiazolidin-3-yl)acetic acid (6d) IR $\left(v_{\max }, \mathrm{KBr}\right) \mathrm{cm}^{-1}: 3472,3418,1736,1690,1612,1520$, $1450,1396,1296,1242,1180,1142,1103,1018 .{ }^{1} \mathrm{H}$ NMR (DMSO-d 6 , 300 MHz, $\delta$ ): 8.776 (s, 1H, Pyrazolyl H) 8.006-7.980 (d, 2H, Ar H, J= $7.8 \mathrm{~Hz}$ ), 7.687 (s, 1H, $=\mathrm{CH}), 7.656-7.544(\mathrm{~m}, 6 \mathrm{H}, \mathrm{Ar} \mathrm{H}), 7.449-7.365(\mathrm{~m}, 1 \mathrm{H}$, Ar H), 4.350 (s, 2H, $\mathrm{NCH}_{2}$ ). MS (ESI+) $m / z 426[\mathrm{M}+\mathrm{H}]$. Anal. Found: C, 57.0; H, 3.4; N, 9.4. $\mathrm{C}_{21} \mathrm{H}_{14} \mathrm{ClN}_{3} \mathrm{O}_{4} \mathrm{~S}$ requires $C$, 57.34; $\mathrm{H}, 3.21 ; \mathrm{N}, 9.55 \%$.

\section{2-((Z)-5-((3-(4-Fluorophenyl)-1-phenyl-1H-pyrazol-4-yl)} methylene)-2, 4-dioxothiazolidin-3-yl)acetic acid (6e) IR $\left(v_{\max }, \mathrm{KBr}\right) \mathrm{cm}^{-1}: 1751,1697,1612,1512,1450,1373$, 1319, 1227, 1149, 1095, 1003. ${ }^{1} \mathrm{H}$ NMR (DMSO-d 6 , 300 $\mathrm{MHz}, \delta): 8.819$ (s, 1H, Pyrazolyl H), 8.048-8.022 (d, 2H, Ar $\mathrm{H}, J=7.8 \mathrm{~Hz}), 7.737-7.711(\mathrm{~m}, 3 \mathrm{H},=\mathrm{CH}$ and $\mathrm{Ar} \mathrm{H})$, 7.607-7.556 (m, 2H, Ar H), 7.455-7.396 (m, 3H, Ar H), 4.369 (s, 2H, $\mathrm{NCH}_{2}$ ). MS (ESI+) $m / z 410[\mathrm{M}+\mathrm{H}]$. Anal. Found: $\mathrm{C}, 59.4 ; \mathrm{H}, 3.5 ; \mathrm{N}, 9.8 . \mathrm{C}_{21} \mathrm{H}_{14} \mathrm{FN}_{3} \mathrm{O}_{4} \mathrm{~S}$ requires C, 59.57; H, 3.33; N, 9.92\%.

2-((Z)-5-((3-(4-Bromophenyl)-1-phenyl-1H-pyrazol-4-yl) methylene)-2, 4-dioxothiazolidin-3-yl)acetic acid (6f) IR $\left(v_{\max }, \mathrm{KBr}\right) \mathrm{cm}^{-1}: 1744,1697,1605,1528,1504,1443$, 1389, 1319, 1242, 1149, 1103, 1003. ${ }^{1} \mathrm{H}$ NMR (DMSO$\left.\mathrm{d}_{6}, 300 \mathrm{MHz}, \delta\right): 8.822$ (s, 1H, Pyrazolyl H), 8.039-8.013 (m, 2H, Ar H), 7.798-7.771 (d, 2H, Ar H, $J=8.1 \mathrm{~Hz}$ ), $7.712(\mathrm{~s}, 1 \mathrm{H},=\mathrm{CH}), 7.634-7.607(\mathrm{~d}, 2 \mathrm{H}, \mathrm{Ar} \mathrm{H}, J=8.1$ $\mathrm{Hz}$ ), 7.581-7.555 (m, 2H, Ar H), 7.460-7.413 (m, 1H, Ar $\mathrm{H}), 4.372\left(\mathrm{~s}, 2 \mathrm{H}, \mathrm{NCH}_{2}\right)$. MS (ESI+) $m / z 470[\mathrm{M}+\mathrm{H}]$. Anal. Found: C, 51.9; H, 2.8; N, 8.5. $\mathrm{C}_{21} \mathrm{H}_{14} \mathrm{BrN}_{3} \mathrm{O}_{4} \mathrm{~S}$ requires $C, 52.08 ; \mathrm{H}, 2.91 ; \mathrm{N}, 8.68 \%$.

2-((Z)-5-((3-(4-Hydroxyphenyl)-1-phenyl-1H-pyrazol-4-yl) methylene)-2, 4-dioxothiazolidin-3-yl)acetic acid (6g) IR $\left(v_{\max }, \mathrm{KBr}\right) \mathrm{cm}^{-1}: 3379,3310,1736,1713,1674,1605$, 1512, 1443, 1404, 1373, 1219, 1142, 1103, 1057, 1003. 
${ }^{1} \mathrm{H}$ NMR (DMSO-d $6,300 \mathrm{MHz}, \delta$ ): 9.886 (bs, 1H, Ph $\mathrm{OH}), 8.753$ (s, 1H, Pyrazolyl H), 8.026-8.000 (d, 2H, Ar $\mathrm{H}, J=7.8 \mathrm{~Hz}), 7.721(\mathrm{~s}, 1 \mathrm{H},=\mathrm{CH}), 7.591-7.540(\mathrm{~m}, 2 \mathrm{H}$, Ar H), 7.476-7.388 (m, 3H, Ar H), 6.960-6.933 (d, 2H, Ar $\mathrm{H}, J=8.1 \mathrm{~Hz}), 4.361\left(\mathrm{~s}, 2 \mathrm{H}, \mathrm{NCH}_{2}\right)$. MS (ESI+) $m / z$ $408[\mathrm{M}+\mathrm{H}]$. Anal. Found: C, 59.7; H, 3.7; N, 9.8. $\mathrm{C}_{21} \mathrm{H}_{15} \mathrm{~N}_{3} \mathrm{O}_{5} \mathrm{~S}$ requires $\mathrm{C}, 59.85 ; \mathrm{H}, 3.59 ; \mathrm{N}, 9.97 \%$.

2-((Z)-5-((3-(4-Nitrophenyl)-1-phenyl-1H-pyrazol-4-yl) methylene)-2, 4-dioxothiazolidin-3-yl)acetic acid (6h) IR $\left(v_{\max }, \mathrm{KBr}\right) \mathrm{cm}^{-1}: 3418,3479,1774,1728,1674,1605$, 1528, 1404, 1350, 1242, 1180, 1142, $11031065 .{ }^{1} \mathrm{H}$ NMR $($ DMSO-d $6,300 \mathrm{MHz}, \delta): 8.887$ (s, 1H, Pyrazolyl H), 8.433-8.404 (d, 2H, Ar H, J=8.7 Hz), 8.066-8.039 (d, 2H, Ar H, J = 8.1 Hz), 7.983-7.954 (d, 2H, Ar H, $J=8.7 \mathrm{~Hz})$, $7.763(\mathrm{~s}, 1 \mathrm{H},=\mathrm{CH}), 7.622-7.571(\mathrm{~m}, 2 \mathrm{H}, \mathrm{Ar} \mathrm{H}), 7.482-$ $7.434(\mathrm{~m}, 1 \mathrm{H}, \mathrm{Ar} \mathrm{H}), 4.384\left(\mathrm{~s}, 2 \mathrm{H}, \mathrm{NCH}_{2}\right) . \mathrm{MS}$ (ESI+) m/ $z 451[\mathrm{M}+\mathrm{H}]$. Anal. Found: C, 55.8; H, 3.0; N, 12.3. $\mathrm{C}_{21} \mathrm{H}_{14} \mathrm{~N}_{4} \mathrm{O}_{6} \mathrm{~S}$ requires $\mathrm{C}, 56.00 ; \mathrm{H}, 3.13 ; \mathrm{N}, 12.44 \%$.

\section{Biological assay}

\section{Test microorganisms}

Four bacteria, S. aureus (MTCC 96), B. subtilis (MTCC 121) (Gram-positive), E. coli (MTCC 1652) and P. aeruginosa (MTCC 741) (Gram-negative) procured from MTCC, Chandigarh and two fungi, $A$. niger and $A$. fla$v u s$, the ear pathogens isolated from the Kurukshetra patients, were used in this study [52].

\section{In vitro antibacterial activity}

The antibacterial activity of synthesized compounds was evaluated by the agar well-diffusion method. All the cultures were adjusted to 0.5 McFarland standard, which is visually comparable to a microbial suspension of approximately $1.5 \times 10^{8} \mathrm{cfu} / \mathrm{mL}$. $20-\mathrm{mL}$ of Mueller Hinton agar medium was poured into each Petri plate and the agar plates were swabbed with $100 \mu \mathrm{L}$ inocula of each test bacterium and kept for 15 min for adsorption. Using sterile cork borer of 8-mm diameter, wells were bored into the seeded agar plates and these were loaded with a $100-\mu \mathrm{L}$ volume with concentration of $4.0 \mathrm{mg} / \mathrm{mL}$ of each compound reconstituted in the dimethylsulphoxide (DMSO). All the plates were incubated at $37^{\circ} \mathrm{C}$ for $24 \mathrm{~h}$. Antibacterial activity of each synthetic compound was evaluated by measuring the zone of growth inhibition against the test organisms with zone reader (Hi Antibiotic zone scale). DMSO was used as a negative control whereas ciprofloxacin was used as a positive control. This procedure was performed in three replicate plates for each organism [53].

\section{Determination of MIC}

$\mathrm{MIC}$ is the lowest concentration of an antimicrobial compound that will inhibit the visible growth of a microorganism after overnight incubation. MIC of the various compounds against bacterial strains was tested through a macro dilution tube method as recommended by NCCLS [54]. In this method, various test concentrations of synthesized compounds were made from 128 to $0.25 \mu \mathrm{g} / \mathrm{mL}$ in sterile tubes no. 1 to $10.100-\mu \mathrm{L}$ sterile Mueller Hinton Broth (MHB) was poured in each sterile tube followed by addition of $200 \mu \mathrm{L}$ test compound in tube 1. Twofold serial dilutions were carried out from the tube no. 1 to the tube no. 10 and excess broth $(100 \mu \mathrm{L})$ was discarded from the last tube no. 10. To each tube, 100 $\mu \mathrm{L}$ of standard inoculums $\left(1.5 \times 10^{8} \mathrm{cfu} / \mathrm{mL}\right)$ was added. Ciprofloxacin was used as control. Turbidity was observed after incubating the inoculated tubes at $37^{\circ} \mathrm{C}$ for $24 \mathrm{~h}$.

\section{In vitro antifungal activity}

The antifungal activity of the synthesized compounds was evaluated by poisoned food technique. The molds were grown on Sabouraud dextrose agar (SDA) at $25^{\circ} \mathrm{C}$ for 7 days and used as inocula. $15 \mathrm{~mL}$ of molten SDA $\left(45^{\circ} \mathrm{C}\right)$ was poisoned by the addition of $100 \mu \mathrm{L}$ volume of each compound having concentration of $4.0 \mathrm{mg} / \mathrm{mL}$, reconstituted in the DMSO, poured into a sterile Petri plate and allowed it to solidify at room temperature. The solidified poisoned agar plates were inoculated at the centre with fungal plugs (8-mm diameter), obtained from the actively growing colony and incubated at $25^{\circ} \mathrm{C}$ for 7 days. DMSO was used as the negative control whereas fluconazole was used as the positive control. The experiments were performed in triplicates. Diameter of the fungal colonies was measured and expressed as percent mycelial inhibition determined by applying the formula [55].

Inhibition of mycelial growth $\%=(d c-d t) / d c \times 100$

where $d c$ average diameter of fungal colony in negative control plates, $d t$ average diameter of fungal colony in experimental plates.

\section{Additional material}

Additional file 1: ${ }^{1} \mathrm{H}$ NMR Spectra .(4a); ${ }^{1} \mathrm{H}$ NMR of ethyl 2-((Z)-2, 4dioxo-5-((1, 3-diphenyl-1H-pyrazol-4-yl)methylene)thiazolidin-3-yl)acetate Additional file 2: ${ }^{1} \mathrm{H}$ NMR Spectra .(4b); ${ }^{1} \mathrm{H}$ NMR of ethyl 2-((Z)-2, 4dioxo-5-((1-phenyl-3-p-tolyl-1H-pyrazol-4-yl)methylene)thiazolidin-3-yl) acetate

Additional file 3: ${ }^{1} \mathrm{H}$ NMR Spectra .(4c); ${ }^{1} \mathrm{H}$ NMR of ethyl 2-((Z)-5-((3-(4methoxyphenyl)-1-phenyl-1H-pyrazol-4-yl)methylene)-2, 4dioxothiazolidin-3-yl)acetate

Additional file 4: ${ }^{1} \mathrm{H}$ NMR Spectra .(4d); ${ }^{1} \mathrm{H}$ NMR of ethyl 2-((Z)-5-((3-(4chlorophenyl)-1-phenyl-1H-pyrazol-4-yl)methylene)-2, 4-dioxothiazolidin-3yl)acetate

Additional file 5: ${ }^{1} \mathrm{H}$ NMR Spectra .(4e); ${ }^{1} \mathrm{H}$ NMR of ethyl 2-((Z)-5-((3-(4fluorophenyl)-1-phenyl-1 H-pyrazol-4-yl)methylene)-2, 4-dioxothiazolidin-3yl)acetate 
Additional file 6: ${ }^{1} \mathrm{H}$ NMR Spectra . (4f); ${ }^{1} \mathrm{H}$ NMR of ethyl 2-((Z)-5-((3-(4 bromophenyl)-1-phenyl-1 H-pyrazol-4-yl)methylene)-2, 4-dioxothiazolidin3-yl)acetate

Additional file 7: ${ }^{1} \mathrm{H}$ NMR Spectra .(4g); ${ }^{1} \mathrm{H}$ NMR of ethyl 2-((Z)-5-((3-(4hydroxyphenyl)-1-phenyl-1 H-pyrazol-4-yl)methylene)-2, 4-dioxothiazolidin3-yl)acetate

Additional file 8: ${ }^{1} \mathrm{H}$ NMR Spectra .(4h)) ${ }^{1} \mathrm{H}$ NMR of ethyl 2-((Z)-5-((3-(4nitrophenyl)-1-phenyl-1H-pyrazol-4-yl)methylene)-2, 4-dioxothiazolidin-3yl)acetate

Additional file 9: ${ }^{1} \mathrm{H}$ NMR Spectra .(5a); ${ }^{1} \mathrm{H}$ NMR of methyl 2-((Z)-2, 4dioxo-5-((1, 3-diphenyl-1 H-pyrazol-4-yl)methylene)thiazolidin-3-yl)acetate

Additional file 10: ${ }^{1} \mathrm{H}$ NMR Spectra .(5b); ${ }^{1} \mathrm{H}$ NMR of methyl 2-((Z)-2, 4dioxo-5-((1-phenyl-3-p-tolyl-1H-pyrazol-4-yl)methylene)thiazolidin-3-yl) acetate

Additional file 11: ${ }^{1} \mathrm{H}$ NMR Spectra .(5c); ${ }^{1} \mathrm{H}$ NMR of methyl 2-((Z)-5-((3(4-methoxyphenyl)-1-phenyl-1H-pyrazol-4-yl)methylene)-2, 4dioxothiazolidin-3-yl)acetate

Additional file 12: ${ }^{1} \mathrm{H}$ NMR Spectra.$(5 \mathrm{~d}) ;{ }^{1} \mathrm{H}$ NMR of methyl 2-((Z)-5-((3(4-chlorophenyl)-1-phenyl-1 H-pyrazol-4-yl)methylene)-2, 4dioxothiazolidin-3-yl)acetate

Additional file 13: ${ }^{1} \mathrm{H}$ NMR Spectra .(5e); ${ }^{1} \mathrm{H}$ NMR of methyl 2-((Z)-5-((3(4-fluorophenyl)-1-phenyl-1H-pyrazol-4-yl)methylene)-2, 4-dioxothiazolidin3-yl)acetate

Additional file 14: ${ }^{1} \mathrm{H}$ NMR Spectra .(5f); ${ }^{1} \mathrm{H}$ NMR of methyl 2-((Z)-5-((3(4-bromophenyl)-1-phenyl-1H-pyrazol-4-yl)methylene)-2, 4dioxothiazolidin-3-yl)acetate

Additional file 15: ${ }^{1} \mathrm{H}$ NMR Spectra.$(5 \mathrm{~g}) ;{ }^{1} \mathrm{H}$ NMR of methyl 2-((Z)-5-((3(4-hydroxyphenyl)-1-phenyl-1H-pyrazol-4-yl)methylene)-2, 4dioxothiazolidin-3-yl)acetate

Additional file 16: ${ }^{1} \mathrm{H}$ NMR Spectra .(5h); ${ }^{1} \mathrm{H}$ NMR of methyl 2-((Z)-5-((3(4-nitrophenyl)-1-phenyl-1H-pyrazol-4-yl)methylene)-2, 4-dioxothiazolidin3-yl)acetate

Additional file 17: ${ }^{1} \mathrm{H}$ NMR Spectra .(6a); ${ }^{1} \mathrm{H}$ NMR of 2-((Z)-2, 4-dioxo-5((1, 3-diphenyl-1H-pyrazol-4-yl)methylene)thiazolidin-3-yl)acetic acid Additional file 18: ${ }^{1} \mathrm{H}$ NMR Spectra .(6b); ${ }^{1} \mathrm{H}$ NMR of 2-((Z)-2, 4-dioxo-5((1-phenyl-3-p-tolyl-1H-pyrazol-4-yl)methylene)thiazolidin-3-yl)acetic acid

Additional file 19: ${ }^{1} \mathrm{H}$ NMR Spectra .(6c); ${ }^{1} \mathrm{H}$ NMR of $2-((\mathrm{Z})-5-((3-(4-$ methoxyphenyl)-1-phenyl-1H-pyrazol-4-yl)methylene)-2, 4-

dioxothiazolidin-3-yl)acetic acid

Additional file 20: ${ }^{1} \mathrm{H}$ NMR Spectra .(6d); ${ }^{1} \mathrm{H}$ NMR of 2-((Z)-5-((3-(4chlorophenyl)-1-phenyl-1H-pyrazol-4-yl)methylene)-2, 4-dioxothiazolidin-3yl)acetic acid

Additional file 21: ${ }^{1} \mathrm{H}$ NMR Spectra .(6e); ${ }^{1} \mathrm{H}$ NMR of 2-((Z)-5-((3-(4fluorophenyl)-1-phenyl-1H-pyrazol-4-yl)methylene)-2, 4-dioxothiazolidin-3yl)acetic acid

Additional file 22: ${ }^{1} \mathrm{H}$ NMR Spectra .(6f); ${ }^{1} \mathrm{H}$ NMR of 2-((Z)-5-((3-(4bromophenyl)-1-phenyl-1H-pyrazol-4-yl)methylene)-2, 4-dioxothiazolidin3-yl)acetic acid

Additional file 23: ${ }^{1} \mathrm{H}$ NMR Spectra .(6g); ${ }^{1} \mathrm{H}$ NMR of 2-((Z)-5-((3-(4hydroxyphenyl)-1-phenyl-1 1 -pyrazol-4-yl)methylene)-2, 4-dioxothiazolidin3-yl)acetic acid

Additional file 24: ${ }^{1} \mathrm{H}$ NMR Spectra .(6h); ${ }^{1} \mathrm{H}$ NMR of $2-((\mathrm{Z})-5-((3-(4-$ nitrophenyl)-1-phenyl-1H-pyrazol-4-yl)methylene)-2, 4-dioxothiazolidin-3yl)acetic acid

\section{Abbreviations}

DMSO: dimethylsulfoxide; MIC: minimum inhibitory concentration; MTCC: microbial-type culture collection; SDA: Sabouraud dextrose agar; TZDs: thiazolidine-2,4-dione.

\section{Acknowledgements}

DKA and PL are thankful to the CSIR and UGC, New Delhi, for providing JRF and SRF, respectively. We are grateful to the Director, SAIF, Punjab University, Chandigarh, for carrying out mass spectrometric analysis. Thanks are due to the CDRl, Lucknow, for carrying out elemental analysis.

\section{Author details}

'Department of Chemistry, Kurukshetra University, Kurukshetra 136119, Haryana, India ${ }^{2}$ Department of Microbiology, Kurukshetra University, Kurukshetra 136119, Haryana, India ${ }^{3}$ Institute of Pharmaceutical Sciences, Kurukshetra University, Kurukshetra 136119, Haryana, India

\section{Competing interests}

The authors declare that they have no competing interests.

Received: 14 July 2011 Accepted: 8 November 2011 Published: 8 November 2011

\section{References}

1. Berber I, Cokmus C, Atalan E (2003) Characterization of Staphylococcus species by SDS-PAGE of whole cell and extracellular proteins. Microbiology 72:42-47. doi:10.1023/A:1022221905449.

2. Bildirici I, Sener A, Tozlu I (2007) Further derivatives of 4-benzoyl-1, 5diphenyl-1H pyrazole-3-carboxylic acid and their antibacterial activities. Med Chem Rev 16:418-426. doi:10.1007/s00044-007-9082-z.

3. Sung WS, Jung HJ, Park K, Kim HS, Lee Ln-S, Lee DG (2007) 2, 5-Dimethyl-4hydroxy-3(2H)-furanone (DMHF): antimicrobial compound with cell cycle arrest in nosocomial pathogens. Life Sci 80:586-591. doi:10.1016/j. Ifs.2006.10.008

4. Aggarwal R, Kumar $V$, Tyagi P, Singh SP (2006) Synthesis and antibacterial activity of some new 1-heteroaryl-5-amino-3H/methyl-4-phenylpyrazoles. Bioorg Med Chem 14:1785-1791. doi:10.1016/j.bmc.2005.10.026.

5. Kumar V, Aggarwal R, Tyagi P, Singh SP (2005) Synthesis and antibacterial activity of some new 1-heteroaryl-5-amino-4-phenyl-3trifluoromethylpyrazoles. Eur J Med Chem 40:922-927. doi:10.1016/j. ejmech.2005.03.021.

6. Kane JL, Hirth BH, Liang B, Gourlie BB, Nahill S, Barsomian G (2003) Ureas of 5-aminopyrazole and 2-aminothiazole inhibit growth of gram-positive bacteria. Bioorg Med Chem Lett 13:4463-4466. doi:10.1016/j. bmcl.2003.09.013.

7. Deohate PP, Deohate JP, Berad BN (2004) Synthesis of some novel 1, 2, 4dithiazolidines and their antibacterial and antifungal activity. Asian J Chem 16:255-260

8. Prakash O, Kumar R, Parkash V (2008) Synthesis and antifungal activity of some new 3-hydroxy-2-(1-phenyl-3-aryl-4-pyrazolyl)chromones. Eur J Med Chem 43:435-440. doi:10.1016/j.ejmech.2007.04.004.

9. Mares D, Romagnoli C, Andreotti E, Manfrini M, Vicentini CB (2004) Synthesis and antifungal action of new tricyclazole analogues. J Agric Food Chem 52:2003-2009. doi:10.1021/jf030695y.

10. Prakash O, Aneja DK, Arora S, Sharma C, Aneja KR (2010) Synthesis and antimicrobial activities of some new 5-((3-(aryl)-1-phenyl-1H-pyrazol-4-yl) methylene)-3-phenylthiazolidine-2, 4-diones. Med Chem Res.

11. Romagnoli C, Mares D, Bruni A, Andreotti E, Manfrini M, Vicentini CB (2001) Antifungal activity of 5 new synthetic compounds vs. Trichophyton rubrum and Epidermophyton floccosum. Mycopathologia 153:129-132

12. Chen H, Li Z, Han Y (2000) Synthesis and fungicidal activity against Rhizoctonia solani of 2-alkyl (alkylthio)-5-pyrazolyl-1, 3, 4-oxadiazoles (thiadiazoles). J Agric Food Chem 48:5312-5315. doi:10.1021/jf991065s

13. Singh N, Sangwan NK, Dhindsa KS (2000) Synthesis and fungitoxic activity of 5-aryl-1-formyl-4, 5-dihydro-3-(2-hydroxyphenyl)-1H-pyrazoles and their complexes. Pest Manag Sci 56:284-288. doi:10.1002/(SICI)1526-4998(200003) 56:33.0.CO;2-M

14. Tanitame A, Oyamada Y, Ofuji K, Fujimoto M, Suzuki K, Ueda T, Tearuchi H, Kawasaki M, Nagai K, Wachi M, Yamagishi J (2004) Synthesis and antibacterial activity of novel and potent DNA gyrase inhibitors with azole ring. Bioorg Med Chem 12:5515-5524. doi:10.1016/.j.bmc.2004.08.010

15. Kees KL, Fitzgerald JJ, Steiner KE, Mattes JF, Mihan B, Tosi T, Moondoro D, McCaleb ML (1996) New potent antihyperglycemic in db/db mice: synthesis 
and structure-activity relationship studies of (4-substituted benzyl) (trifloromethyl)pyrazoles and -pyrazolones. J Med Chem 39:3920-3928. doi:10.1021/jm960444z

16. Meazza G, Bettarini F, Porta PL, Piccardi P, Signorini E, Portoso D, Fornara L (2004) Synthesis and herbicidal activity of novel heterocyclic protoporphyrinogen oxidase inhibitors. Pest Manag Sci 60:1178-1188. doi:10.1002/ps.923.

17. Waldrep TW, Beck JR, Lynch MP, Wright FL (1990) Synthesis and herbicidal activity of 1-aryl-5-halo- and 1-aryl-5-(trifluoromethyl)-1H-pyrazole-4carboxamides. J Agric Food Chem 38:541-544. doi:10.1021/jf00092a045.

18. Park H-J, Lee K, Park S-J, Ahn B, Lee J-C, Cho HY, Lee K-I (2005) Identification of antitumor activity of pyrazole oxime ethers. Bioorg Med Chem Lett 15:3307-3312. doi:10.1016/j.bmcl.2005.03.082.

19. Hatheway GJ, Hansch C, Kim KH, Milstein SR, Schmidt CL, Smith RN (1978) Antitumor 1-(X-aryl)-3, 3-dialkyltriazenes. 1. Quantitative structure-activity relationships vs. L1210 Leukemia in Mice. J Med Chem 21:563-574. doi:10.1021/jm00204a012.

20. Katayama H, Oshiyama T (1997) Preparation and bioactivity of pyrazole derivatives as potential cross-linking agent. Can J Chem 75:913-919

21. Manfredini S, Bazzanini R, Baraaldi PG, Guameri M, Simoni D, Marongiu ME, Pani A, Tramontano E, Colla PL (1992) Pyrazole-related nucleosides. Synthesis and antiviral/antitumor activity of some substituted pyrazole and pyrazolo[4,3-d]-1,2,3-triazin-4-one nucleosides. J Med Chem 35:917-924. doi:10.1021/jm00083a017.

22. Wustrow DJ, Rubin CR, Knobelsdorf JA, Akunne $H$, Mackenzie DR, Pugsley TA, Zoski KT, Heffner TG, Wise LD (1998) Pyrazolo[1, 5-a]pyrimidine CRF-1 receptor antagonists. Bioorg Med Chem Lett 8:2067-2070. doi:10.1016/S0960-894X(98)00372-2.

23. Habeeb AG, Rao PNP, Knaus EE (2001) Design and synthesis of celecoxib and rofecoxib analogues as selective cyclooxygenase-2 (COX-2) inhibitors: replacement of sulfonamide and methylsulfonyl pharmacophores by an azido bioisostere. J Med Chem 44:3039-3042. doi:10.1021/jm010153c.

24. Martel AM, Graul A, Rabbaseda X, Castaner R (1997) Slidenafil: treatment of erectile dysfunction, phosphodiesterase $V$ inhibitor. Drugs Future 22:138-143

25. Yoshioka T, Fujita T, Kanai T, Aizava Y, Kurumada T, Hasegava K, Horikoshi H (1989) Studies on hindered phenols and analogues. 1. Hypolipidemic and hypoglycaemic agents with ability to inhibit lipid peroxidation. J Med Chem 32:421-428. doi:10.1021/jm00122a022.

26. Lee HW, Kim BY, Ahn JB, Kang SW, Lee JH, Shin JS, Ahn SK, Lee SJ, Yoon SS (2005) Molecular design, synthesis, and hypoglycaemic and hypolipidemic activities of novel pyrimidine derivatives having thiazolidinedione. Eur J Med Chem 40:862-874. doi:10.1016/j.ejmech.2005.03.019.

27. Bozdag-Dundar O, Verspohl EJ, Das-Evcimen N, Kaup RM, Bauer K, Sarikaya M, Evranos B, Ertan R (2008) Synthesis and biological activity of some new flavonyl-2, 4-thiazolidinediones. Bioorg Med Chem 16:6747-6751. doi:10.1016/j.bmc.2008.05.059.

28. Eun JS, Kim KS, Kim HN, Park SA, Ma T, Lee KA, Kim DK, Kim HK, Kim IS, Jung YH, Zee OP, Yoo DJ, Kwak YG (2007) Synthesis of psoralen derivatives and their blocking effect of hkv1.5 channel. Arch Pharm Res 30:155-160. doi:10.1007/BF02977688.

29. Mori M, Takagi M, Noritake C, Kagabu S (2008) 2, 4-Dioxo-1, 3-thiazolidine derivatives as a lead for new fungicides. J Pestic Sci 33:357-363. doi:10.1584/jpestics.G08-15.

30. Sahu SK, Banerjee M, Mishra SK, Mohanta RK (2007) Synthesis, partition coefficient and antibacterial activity of $3^{\prime}$-phenyl(substituted)- $6^{\prime}$-aryl-2' $(1 \mathrm{H})$ cis-3', 3'a-dihydrospiro[3-H-indole-3, 5'-pyrazolo (3', 4'-d)-thiazolo-2-(1H)ones]. Acta Pol Pharm Drug Res 64:121-126

31. Dwivedi C, Gupta TK, Parmar SS (1972) Substituted thiazolidones as anticonvulsants. J Med Chem 15:553-554. doi:10.1021/jm00275a031.

32. Verma A, Saraf SK (2008) 4-Thiazolidinone-a biological active scaffold. Eur J Med Chem 43:897-905. doi:10.1016/j.ejmech.2007.07.017.

33. Ceriello A (2008) Thiazolidinediones as anti-inflammatory and antiatherogenics. Diab Met Res Rev 24:14-26. doi:10.1002/dmrr.790.

34. Bozdag-Dundar O, Ozgen O, Mentese A, Altanlar N, Atli O, Kendib E, Eetana R (2007) Synthesis and antimicrobial activity of some new thiazolyl thiazolidine-2, 4-dione derivatives. Bioorg Med Chem 15:6012-6017. doi:10.1016/j.bmc.2007.06.049.

35. Brooke EW, Davies SG, Mulvaney AW, Okada M, Pompeo F, Sim E, Vickers RJ, Westwood IM (2003) Synthesis and in vitro evaluation of novel small molecule inhibitors of bacterial arylamine N-acetyltransferases (NATs). Bioorg Med Chem Lett 13:2527-2530. doi:10.1016/S0960-894X(03)00484-0.

36. Tuncbilek M, Altanlar N (1999) Synthesis and antimicrobal evaluation of some 3-(substituted phenacyl)-5-[4'-(4H-oxo-1-benzopyran-2-yl)benzylidene]-2, 4-thiazolidinediones. IL Farmaco 54:475-478. doi:10.1016/ S0014-827X(99)00054-3.

37. Tuncbilek M, Bozda O, Bozdag-Dundar O, Ayhan-Kilcigil G, Ceylan M, Waheed A, Verspohl EJ, Ertan R (2003) Synthesis and hypoglycemic activity of some substituted flavonyl thiazolidinedione derivatives-fifth communication: flavonyl benzyl substituted 2, 4-thiazolidinediones. IL Farmaco 58:79-83. doi:10.1016/S0014-827X(02)01241-7.

38. Pomel V, Klicic J, Covini D, Church DD, Shaw JP, Roulin K, BurgatCharvillon F, Valognes D, Camps M, Chabert C, Gillieeron C, Francon B, Perrin D, Leroy D, Gretener D, Nichols A, Vitte PA, Carboni S, Rommel C, Schwarz MK, Ruckle T (2006) Furan-2-ylmethylene thiazolidinediones as novel, potent, and selective inhibitors of phosphoinositide-3-kinase $\gamma$. J Med Chem 49:3857-3871. doi:10.1021/jm0601598.

39. Bozdag-Dundar O, Evranos B, Das-Evcimen N, Sarikaya M, Ertan R (2008) Synthesis and aldose reductase inhibitory activity of some new chromonyl2, 4-thiazolidinediones. Eur J Med Chem 43:2412-2417. doi:10.1016/j. ejmech.2008.01.004.

40. Youssef AM, White MS, Villanueva EB, El-Ashmawy IM, Klegeris A (2010) Synthesis and biological evaluation of novel pyrazolyl-2, 4thiazolidinediones as anti-inflammatory and neuroprotective agents. Bioorg Med Chem 18:2019-2028. doi:10.1016/j.bmc.2010.01.021.

41. Prakash O, Kumar M, Kumar R, Sharma C, Aneja KR (2010) Hypervalent lodine(III) mediated synthesis of novel unsymmetrical 2, 5-disubstituted 1, 3, 4-oxadiazoles as antibacterial and antifungal agents. Eur J Med Chem 45:4252-4257. doi:10.1016/j.ejmech.2010.06.023.

42. Prakash O, Kumar R, Sehrawat $R$ (2009) Synthesis and antibacterial activity of some new 2, 3-dimethoxy-3-hydroxy-2-(1-phenyl-3-aryl-4-pyrazolyl) chromanones. Eur J Med Chem 44:1763-1767. doi:10.1016/j. ejmech.2008.03.028.

43. Kumar R, Nair RR, Dhiman SS, Sharma J, Prakash O (2009) Organoiodine(III)mediated synthesis of 3-aryl/heteroaryl-5, 7-dimethyl-1, 2, 4-triazolo[4, 3-c] pyrimidines as antibacterial agents. Eur J Med Chem 44:2260-2264. doi:10.1016/j.ejmech.2008.06.004.

44. Prakash O, Aneja DK, Wadhwa D, Kumar R (2011) A facile synthesis of novel dihydroindeno[1, 2-e][1, 2, 4]triazolo[3, 4-b][1, 3, 4]thiadiazines using HTIB. J Heterocycl Chem. doi:10.1002/jhet.815

45. Kira MA, Abdel-Rahman MO, Gadalla KZ (1969) The Vilsmeier-Haack reaction-III cyclization of hydrazones to pyrazoles. Tetrahedron Lett 10:109-110. doi:10.1016/S0040-4039(01)88217-4.

46. Bernard M, Hulley E, Molenda H, Stochla K, Wrzeciono U (1986) Azoles. 17. Beta-(4-pyrazol)acrylic and propionic acids and their anti-inflammatory activity. Pharmazie 41:560-562

47. De Luca L, Giacomelli G, Masala S, Porchddu A (2004) A mild procedure for the preparation of 3-aryl-4-formylpyrazoles. Synlett 13:2299-2302

48. Xie Y, Liu Y, Gong G, Rinderspacher A, Deng S-X, Smith DH, Toebben U, Tzilianos E, Branden L, Vidovic D, Chung C, Schurer S, Tautz L, Landry DW (2008) Discovery of a novel submicromolar inhibitor of the lymphoid specific tyrosine phosphatase. Bioorg Med Chem Lett 18:2840-2844. doi:10.1016/j.bmcl.2008.03.079.

49. Pattana SR, Kekareb P, Patlic A, Nikaljec A, Kitturd BS (2009) Studies on the synthesis of novel 2, 4-thiazolidinedione derivatives with antidiabetic activity. Iran J Pharm Sci 5:225-230

50. El-Deeb IM, Bayoumi SM, El-Sherbeny MA, Abdel-Aziz AA-M (2010) Synthesis and antitumor evaluation of novel cyclic arylsulfonylureas: ADME-T and pharmacophore prediction. Eur J Med Chem 45:2516-2530. doi:10.1016/j. ejmech.2010.02.038.

51. Kumar BRP, Soni M, Kumar SS, Singh K, Patil M, Baig RBN, Adhikari L (2011) Synthesis, glucose uptake activity and structure-activity relationships of some novel glitazones incorporated with glycine, aromatic and alicyclic amine moieties via two carbon acyl linker. Eur J Med Chem 46:835-844. doi:10.1016/j.ejmech.2010.12.019.

52. Aneja KR, Sharma C, Joshi R (2010) Fungal infection of the ear: a common problem in the north eastern part of Haryana. Int J Pediatr Otorhinolaryngol 74:604-607. doi:10.1016/j.ijporl.2010.03.001.

53. Ahmad I, Beg AZ (2001) Antimicrobial and phytochemical studies on 45 Indian medicinal plants against multi-drug resistant human pathogens. J Ethnopharmacol 74:113-123. doi:10.1016/S0378-8741(00)00335-4. 
54. Villanova PA (2000) NCCLS, National Committee for Clinical Laboratory Standards.

55. Al-Burtamami SKS, Fatope MO, Marwah RG, Onifade AK, Al-Saidi SH (2005)

Chemical composition, antibacterial and antifungal activities of the essential

oil of halophyllum tuberculatum from Oman. J Ethnopharmacol 96:107-112. doi:10.1016/j.jep.2004.08.039.

doi:10.1186/2191-2858-1-15

Cite this article as: Aneja et al: Synthesis of new pyrazolyl-2, 4-

thiazolidinediones as antibacterial and antifungal agents. Organic and

Medicinal Chemistry Letters 2011 1:15.

\section{Submit your manuscript to a SpringerOpen ${ }^{\circ}$ journal and benefit from:}

- Convenient online submission

- Rigorous peer review

- Immediate publication on acceptance

- Open access: articles freely available online

- High visibility within the field

- Retaining the copyright to your article

Submit your next manuscript at $\gg$ springeropen.com 